\title{
Maculopathie causée par le polysulfate de pentosan
}

\author{
Daniel Rosenberg BSc, David Sarraf MD, Varun Chaudhary MD
}

Citation : CMAJ 2021 May 3;193:E645. doi : 10.1503/cmaj.201900-f

Voir la version anglaise de l'article ici : www.cmaj.ca/lookup/doi/10.1503/cmaj.201900
1 Le polysulfate de pentosan est le principal traitement de la douleur vésicale associée à La cystite interstitielle
La cystite interstitielle est un trouble chronique de la vessie qui cause une douleur pelvienne et des mictions fréquentes ou urgentes, avec une prévalence estimée de $2,7 \%-6,5 \%$ chez les femmes adultes ${ }^{1}$. Le polysulfate de pentosan peut agir comme un tampon entre l'épithélium vési- cal et les irritants; il s'agit du seul médicament oral approuvé par Santé Canada pour traiter la cystite interstitielle ${ }^{1}$. Il est généralement administré par voie orale en doses de $100 \mathrm{mg}$ prises 3 fois par jour.

2

La maculopathie est associée à une utilisation prolongée du polysulfate de pentosan

Une vaste étude rétrospective a révélé une augmentation de la probabilité de maladie maculaire après une exposition cumulative de 7 ans au polysulfate de pentosan (rapport de cotes 1,41; intervalle de confiance à $95 \% 1,09-1,83)^{2}$. Une étude transversale de 50 patients ayant été traités au polysulfate de pentosan a montré que $15 \%-20 \%$ d'entre eux manifestaient des signes de toxicité maculaire décelables à l'imagerie rétinienne. La prévalence de la maladie maculaire pouvait atteindre $50 \%$ chez les patients dont la dose cumulative dépassait $1500 \mathrm{~g}^{3}$.

La maculopathie causée par le polysulfate de pentosan peut s'apparenter à la

dégénérescence maculaire liée à l'âge

La maculopathie causée par le polysulfate de pentosan est associée à des difficultés de lecture et à des troubles de la vision dans des conditions de faible luminosité. Les patients qui en sont atteints obtiennent souvent des diagnostics de maladies plus fréquentes, comme la dégénérescence maculaire liée à l'âge ${ }^{2}$.

La maladie maculaire peut poursuivre sa progression malgré l'arrêt du polysulfate de pentosan

On croit que la pathogenèse de la maculopathie liée au polysulfate de pentosan comprend une perturbation de la matrice extracellulaire entourant les photorécepteurs ou une toxicité directe de l'épithélium pigmentaire de la rétine $\mathrm{e}^{2-4}$. On ne sait pas si l'évolution de la maladie est modifiée par l'interruption de la prise du médicament ${ }^{4}$. Des études de cas ont documenté la progression de la maculopathie jusqu'à 6 ans après la cessation de la prise du polysulfate de pentosan ${ }^{5}$.

Les patients exposés au polysulfate de pentosan qui rapportent des troubles de la vision devraient subir un dépistage ophtalmique

Les prescripteurs de polysulfate de pentosan et les fournisseurs de soins primaires devraient

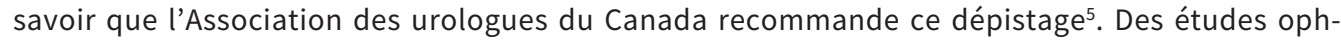
talmiques récentes recommandent une ophtalmoscopie et une prise d'images initiales, particulièrement chez les patients dont l'exposition cumulative au polysulfate de pentosan dépasse $500 \mathrm{~g}$, mais davantage de données probantes sont nécessaires pour affiner les lignes directrices de dépistage ${ }^{3,5}$. 


\section{Références}

1. Cox A, Golda N, Nadeau G, et al. CUA guideline: Diagnosis and treatment of interstitial cystitis/bladder pain syndrome. Can Urol Assoc J 2016;10:E136-55.

2. Jain N, Li AL, Yu Y, et al. Association of macular disease with long-term use of pentosan polysulfate sodium: findings from a US cohort. Br J Ophthalmol 2020;104:1093-7.

3. Wang D, Au A, Gunnemann F, et al. Pentosan-associated maculopathy: prevalence, screening guidelines, and spectrum of findings based on prospective multimodal analysis. Can J Ophthalmol 2020;55:116-25.

4. Yusuf IH, Charbel Issa P, Lotery AJ. Pentosan polysulfate maculopathy - prescribers should be aware. JAMA Ophthalmol 2020;138: 900-2.

5. Doiron RC, Bona M, Nickel JC. Possible drug-induced, vision-threatening maculopathy secondary to chronic pentosan polysulfate sodium (Elmiron ${ }^{\circledast}$ ) exposure. Can Urol Assoc J 2020;14:10-1.

Intérêts concurrents : Varun Chaudhary a siégé aux comités consultatifs de Novartis et de Bayer et a été consultant pour ces entreprises. Il touche des subventions de recherche parrainées par des chercheurs et participe à des essais cliniques financés par Novartis, Bayer et Allergan. David Sarraf a été consultant pour Amgen, Bayer, Genentech, Novartis et Optovue, et il reçoit des subventions de recherche d'Amgen, Genentech, Heidelberg Engineering, Optovue, Regeneron et Topcon. Aucun autre intérêt concurrent n'a été déclaré.

Cet article a été révisé par des pairs.

Affiliations : Faculté de médecine Michael G. DeGroote (Rosenberg), Université McMaster, Hamilton, Ont.; Institut d'ophtalmologie Stein (Sarraf), Faculté de médecine David Geffen, Université de Californie à LoS Angeles; Greater Los Angeles Veterans Administration Healthcare Center (Sarraf), Los Angeles, Calif.; Division d'ophtalmologie (Chaudhary), Département de chirurgie et Département des méthodes, des données et de l'incidence de la recherche en santé (Chaudhary), Université McMaster; Hôpital St. Joseph de Hamilton et Université McMaster (Chaudhary), Hamilton, Ont.

Propriété intellectuelle du contenu : Il s'agit d'un article en libre accès distribué conformément aux modalités de la licence Creative Commons Attributions (CC BY-NC-ND 4.0), qui permet l'utilisation, la diffusion et la reproduction dans tout médium à la condition que la publication originale soit adéquatement citée, que l'utilisation se fasse à des fins non commerciales (c.-à-d. recherche ou éducation) et qu'aucune modification ni adaptation n'y soit apportée. Voir : https://creativecommons.org/licenses/by-nc-nd/4.0/deed.fr.

Correspondance : Varun Chaudhary, vchaudh@mcmaster.ca 